\title{
EDUCACIÓN A DISTANCIA Y FORMACIÓN DOCENTE. RETOS Y DESAFÍOS EN LOS POSTGRADOS DE LA UNIVERSIDAD NACIONAL DE ROSARIO
}

\author{
Susana Copertari (UNR)* \\ Natalia Sgreccia (UNR-CONICET)** \\ Yanina N. Fantasía (UNR)
}

\section{Resumen}

El trabajo que presentamos se enmarca en un Proyecto de Investigación Interdisciplinario (Pol148/10) "Política (s) académica(s) sobre carreras de postgrado y formación docente en educación a distancia en la Universidad Nacional de Rosario", radicado en la Secretaría de Investigación y Postgrado de la Facultad de Ciencia Política y RRII (Universidad Nacional de Rosario), sobre una población integrada por Facultades donde se dictan carreras de postgrado en el marco de la Educación a Distancia.

El objetivo fundamental consiste en analizar crítica y reflexivamente cuál es la(s) política(s) académica(s) y de gestión de nuestra Universidad en materia de educación a distancia, ya sea virtual o semipresencial. Desde la tecnología educativa se intenta indagar acerca de sus implicancias en la formación docente de cada unidad académica.

Acercamos un análisis sobre el estado actual de los conocimientos sobre el tema, como etapa inicial de la investigación. En éste concurren los aportes teóricos de Litwin (1997, 2000, 2001, 2006, 2008), Fainholc (1999, 2000), Mena (2007), Rama (2006, 2007), Morin (1997, 2000). Metodológicamente se sustenta en una lógica cualicuantitativa, con la aplicación del método comparativo continuo (Bolsegui y Fuguet

\footnotetext{
* Magister y Especialista en Docencia Universitaria. Universidad Tecnológica Nacional. Licenciada en Ciencia Política. Facultad de Ciencia Política y RR.II. Universidad Nacional de Rosario.

* Magíster en Didácticas Específicas. Universidad Nacional del Litoral. Profesora en Matemática. Facultad de Ciencias Exactas, Ingeniería y Agrimensura. Universidad Nacional de Rosario. Doctoranda en Educación (UNR), con beca de CONICET.

*** Licenciada y Profesora en Ciencias de la Educación. Facultad de Humanidades y Artes. Universidad Nacional de Rosario. Maestranda de la Maestría en Tecnología Educativa. Universidad de Buenos Aires.
} 
Smith, 2006) y las técnicas del cuestionario, entrevista y análisis documental (campo empírico), para una posterior triangulación.

\section{Palabras clave}

Política(s) académica(s) - Formación docente - Educación a distancia - Gestión del conocimiento - Tecnología educativa.

\section{Summary}

This paper is framed in an Inter-disciplinarian Research Project (Pol148/10) called "Academic Politic(s) about postgraduate careers and training teachers in distance education in the National University of Rosario", which concerns of the Secretary of Research and Postgraduate of the Faculty of Politic Science and International Relationships (National University of Rosario). The population of the research samples is constituted by the Faculties where distance education in postgraduate careers exists.

The main objective is to analyze, by a critical and reflective way, which is the academic politic(s) and the management of our University regarding to distance education, including virtual and semi-presence modalities. Throughout the educative technology, we inquire into its implications in the training teachers of each Faculty.

We approach an analysis of the current advance of the knowledge of the research topic, as an initial stage of the whole project. In it, we articulate the theoretical contributions of Litwin (1997, 2000, 2001, 2006, 2008), Fainholc (1999, 2000), Mena (2007), Rama $(2006,2007)$, Morin $(1997,2000)$. Methodologically, the perspective is qualitative and quantitative, with the application of the continuous comparative method (Bolsegui and Fuguet Smith, 2006) and the techniques of questionnaire, interview and documentary analysis (empirical research field), for a later triangulation.

\section{Keywords}

Academic politic(s) - Training teachers - Distance education - Management of the knowledge - Educative technology.

\section{Introducción}

El Proyecto ha sido presentado en la Facultad de Ciencia Política y Relaciones Internacionales de la Universidad Nacional de Rosario (UNR).

La problemática que nos preocupa tiene que ver con que la UNR ha venido teniendo un desarrollo lento en materia de Política(s) Académica(s) en materia de Educación a Distancia (EAD) en carreras de postgrado. Sólo en algunas carreras se viene implementando esta modalidad en el cursado de algunos seminarios en forma virtual o semipresencial (bimodales/híbridos).

Partimos de algunos supuestos al iniciar esta investigación, afirmando que la UNR aún no ha terminado de trazar políticas académico-institucionales sobre la implementación y acreditación de carreras de postgrado a distancia. 
El Campus Virtual de la UNR ha presentado un Proyecto de ordenanza para su institucionalización que aún no ha sido aprobado.

La necesidad de desarrollar Programas de Formación Docente continua se transforma, de este modo, en una necesidad imperiosa en los próximos años, para brindar a los profesionales-docentes de la UNR un conjunto de programas sostenidos en este campo del conocimiento, como así también, de las prácticas educativas en EAD.

Los nuevos escenarios educativos, con el crecimiento de las modalidades no presenciales (a distancia y virtual), en las universidades nacionales, viene teniendo un crecimiento acelerado en el campo de la Educación Superior en nuestro país y en la Región, donde la UNR debe formar parte.

La incorporación creciente de las Tecnologías de la Información y la Comunicación (TICs), herramientas de producción, transporte y comunicación de contenidos en la educación presencial y a distancia, exigen de los docentes el desarrollo de nuevas estrategias educativas para la apropiación social de conocimientos, que demandan nuevos desempeños y niveles de comprensión, como así también, la adecuación de las carreras tradicionales a las exigencias que requiere la educación del Siglo XXI.

La educación mediada por entornos virtuales orientada al aprendizaje activo, reflexivo, crítico y cada vez más autónomo exige a los docentes de nuevas capacidades comunicativas en el marco de los "nuevos lenguajes" y de un enfoque innovador de la enseñanza y el aprendizaje desde un pensamiento complejo (Morin, 2000). Crece imperiosamente la necesidad de que los docentes -especialmente en los postgrados- dominen herramientas informáticas y de comunicación, que les posibiliten desenvolverse con experticia en estos nuevos contextos.

Las instituciones educativas están transformando sus carreras y cursos con modalidades total o parcialmente no presenciales. Las mismas deben ser analizadas desde la perspectiva de la tecnología educativa, para incorporar su vasto espectro, en la utilización de las "Nuevas Tecnologías" (NT) con un sentido pedagógico.

\section{Objetivos del Proyecto}

General:

- Analizar crítica y reflexivamente la(s) política(s) académica(s) de la UNR en materia de EAD, en carreras de Postgrado, y sus implicancias en la formación docente al interior de cada Unidad académica.

Específicos:

- Indagar acerca de cuáles son las Facultades de la UNR donde se cursan carreras de postgrado a distancia, mediante un rastreo témporoespacial. 
- Seleccionar y sistematizar comparativa y comprensivamente los postgrados a distancia conforme a: Especializaciones, Maestrías, Doctorados y el impacto de las TICs en dichos Programas.

- Reflexionar críticamente en cada Unidad Académica localizada sobre: los distintos Programas de EAD, el diseño de los materiales didácticos, las acciones tutoriales, el sistema de gestión académico-institucional, la gestión del conocimiento, la cultura académica, el estilo de enseñanza y aprendizaje, la utilización de las TICs, en el marco de la complejidad y la tecnología educativa.

- Realizar un aporte a las políticas académicas de la UNR en relación al campo del conocimiento científico en materia de postgrados con EAD y formación docente desde la perspectiva de la tecnología educativa.

\section{Acerca de la Metodología}

Estamos transitando una etapa inicial del Proyecto, por lo cual esta investigación se está llevando a cabo a través de un rastreo de las carreras de postgrado a distancia en nuestra Universidad y a las políticas que las sustentan, en documentos y proyectos institucionales por la Web, y sobre una revisión de la literatura existente sobre el tema.

En razón a la complejidad del problema a investigar, en primer lugar nos propusimos recuperar experiencias de EAD en carreras de postgrado de la UNR y, a partir de allí, desentrañar si existe(n) política(s) académica(s) en esta Universidad, en el marco de una planificación estratégica en materia de "ofertas" de EAD, con respecto a este tipo de educación y a Programas de Formación Docente continua para llevarlas adelante resguardando la calidad educativa.

Luego, en una segunda etapa, indagaremos acerca de los distintos enfoques, concepciones, líneas de investigación, propuestas y experiencias de los docentes que participan o participaron en las mismas. Por último, intentaremos realizar aportes que contribuyan, tanto a la formación de profesores en el área de EAD, como a las políticas académicas sobre dicha modalidad en la UNR.

El carácter exploratorio-descriptivo e interpretativo-crítico de la problemática planteada nos ha llevado a optar por una estrategia metodológica cualicuantitativa, desde un enfoque antropológico y socio-cultural (Guber, 2002), por lo cual se enfatizará la perspectiva cualitativa.

La misma nos compromete a asumir el objeto de estudio desde una mirada compleja, para poder comprender las incertidumbres (Morin, 1995). Para ello realizaremos en la etapa final de la recopilación de la información, una triangulación intermetodológica (Denzin, 1978), donde se combinarán fundamentalmente tres técnicas: la entrevista semiestructurada, las focalizadas y abiertas en profundidad y el análisis documental, para alcanzar una compren- 
sión interpretativa y crítica de las prácticas de enseñanza en cada una de las experiencias, utilizando el método comparativo continuo.

Si lo requiere el despliegue investigativo tenemos planteado utilizar, a modo complementario, el cuestionario (vía electrónica) y la estadística, sólo en aquellos casos en que necesitemos ilustrar algunos resultados que pudieran facilitar la articulación (dato/información/comprensión), con el abordaje cualitativo y el corpus teórico.

Adherimos a Valles (1999, citado por Bolsegui y Fuguet Smith, 2006), en que la metodología cualitativa tiene una lógica que sigue un proceso donde, a partir de una experiencia, se trata de interpretar el contexto bajo diversos puntos de vista. Su diseño está abierto a la invención (creatividad), la obtención de datos al descubrimiento y el análisis a la interpretación.

Tal como plantean Bolsegui y Fuguet Smith (2006), siguiendo a Rodríguez (1999), la investigación se desarrollará con las siguientes fases:

a) Diseño: descripción del Diseño General de la Investigación, y de algunos aspectos del proceso de recolección de la información. Se especifican los tipos de informantes claves seleccionados, así como la naturaleza del método empleado (entrevista cualitativa).

b) Recolección, construcción y análisis: Se explica que tanto la categorización, como el análisis e interpretación son actividades conjuntas, estrechamente vinculadas entre sí.

c) Teorización y construcción del modelo: Se argumenta que la investigación avanza desde una conceptuación preliminar (denominada modelo inicial) que adquiere mayor sentido y profundidad en fases posteriores (modelos intermedios) hasta llegar a una conceptuación final que se presenta como modelo final (modelo conceptual).

d) Categorización, análisis e interpretación: En el ámbito de la investigación cualitativa, uno de los aspectos más álgidos es el análisis. Pareciera que en cuanto a los procedimientos de recogida de información existe mayor acuerdo, prolíficas fuentes de consultas, pero en cuanto al análisis aún es insuficiente. Este proceso exige una inmersión total en el material protocolar a ser estudiado, desnudar las ideas, construir, deconstruir y volver a integrar nuevamente.

\section{Algunos avances en la investigación}

En relación al estado actual de los conocimientos sobre el tema podemos decir que este Proyecto es de reciente iniciación (Pol 148, 2010-2013) y los resultados que aquí presentamos fueron sistematizados como resultado de una búsqueda por la Web y sobre material impreso.

En cuanto a la información disponible por la Web y en los materiales impresos hemos podido rescatar lo siguiente, en primera instancia que la UNR 
cuenta con 12 (doce) Facultades. En las mismas se desarrollan 66 carreras de grado, 124 carreras de postgrado, 15 tecnicaturas, 53 títulos intermedios, 26 títulos por articulación con el sistema de Educación Superior no Universitario y 32 postítulos.

A continuación presentamos un primer relevamiento, a partir de la información online, sobre las carreras de postgrado que se ofrecen en la actualidad en la UNR.

- En la Facultad de Arquitectura, Planeamiento y Diseño se ofrecen Doctorados de Arquitectura con modalidad presencial y Carreras de Especialización presenciales.

- La Facultad de Ciencia Política y Relaciones Internacionales ofrece Doctorados y Maestrías presenciales. En cuanto a las Carreras de Especialización: "Comunicación Digital Interactiva", es presencial y dispone de un Blog; "Estudios Políticos" tiene modalidad presencial con una página Web y Campus Virtual; "Gestión Estratégica de Organizaciones Públicas" se cursa en forma presencial, sin cohorte en 2009.

- En la Facultad de Ciencias Agrarias, los Doctorados, Maestrías y Especializaciones son con modalidad presencial. Se ofrece una Actualización a Distancia en Bioquímica Clínica. Además se consignan cursos de Postgrado cuya modalidad de cursado no se especifica.

- En la Facultad de Ciencias Económicas y Estadísticas, los Doctorados son de carácter presencial. En la Secretaría de Postgrado y Formación Docente hemos podido constatar que en los Doctorados en Administración, en Contabilidad, y en Economía, sólo el "Seminario de Econometría Avanzada" se dicta con Modalidad semipresencial.

- La Facultad de Humanidades y Artes ofrece Doctorados y Maestrías en forma presencial, siendo la Maestría en Artes con modalidad presencial e incluye algunas experiencias semipresenciales. Al respecto, una de nuestras informantes-clave, nos comentó:

(...) las materias que yo dicto en la escuela de música, que son de audio perceptiva uno y dos, las tengo también en comunidades. O sea, mis alumnos acceden a estos materiales también desde el campus. Yo los mando partituras, les mando música (...) consideré importante incluir la modalidad virtual (...) porque hay muchos alumnos de afuera, y (...) uno tiene más comunicación cuando queda conectado desde un sitio. (...). Entonces cualquier necesidad, cualquier pregunta, porque una vez que el alumno ya aprobó la materia o la cursó necesita hacer muchas preguntas (...). Nosotros también aprovechamos para hacer foros de discusión o aprovechamos para informar sobre congresos (Entrevista, 28 de Abril de 2010). 
- La Facultad de Odontología cuenta con Doctorados y Maestrías con modalidad presencial.

- La Facultad de Ciencias Médicas posee Doctorados, Maestrías y Especializaciones. Sólo hemos podido encontrar hasta el momento que a partir de una publicación en el Periódico Digital de la UNR (Universitas), la UNR lanzó un sitio web completo sobre Clínica Médica, en el año 2005:

La Primera Cátedra de Clínica Médica y la carrera de Especialización en Clínica Médica de la (UNR) ya cuentan con un sitio web que brinda información a estudiantes, docentes, profesionales y público en general (...) Este sitio tiene múltiples utilidades. Una de ellas es ofrecer tanto a los alumnos de grado como de postgrado toda la información académica, funcionando como un transparente virtual, donde el alumno puede obtener información relacionada con la Cátedra y/o la Carrera de Posgrado. La página presenta notas editoriales sobre diversos temas médicos y en la sección denominada Revista de Revistas, los docentes comentan artículos de distintas publicaciones de actualidad y de gran utilidad para el clínico. El Profesor Titular coordina la sección de Seminarios centrales y AnátomoClínicos del Servicio de Clínica Médica del Hospital Provincial del Centenario (sede de la cátedra). Previamente a la realización de cada seminario se publica el caso que va a ser analizado y posteriormente, su discusión, acompañada de imágenes relevantes (...) en "La imagen del mes", se exponen contenidos visuales de casos clínicos aportando una herramienta notable en el aprendizaje. También se puede tener acceso a la sección "Medicina y Cultura", en la que se publican trabajos relacionados con distintas expresiones artísticas como literatura, música, pintura, filosofía e historia (...). Se encuentra en desarrollo un índice de Guías, Consensos y Recomendaciones de las Sociedades Científicas de referencia acerca de las principales patologías de la Medicina Interna, proyectos de cursos online y foros de discusión de casos clínicos (Universitas, 2005).

- La Facultad de Ciencias Veterinarias ofrece Doctorados y Maestrías en forma presencial. En cuanto a las Carreras de Especialización, no se especifica la modalidad de cursado en la Web.

- En la Facultad de Derecho tanto los Doctorados, las Maestrías como las Carreras de Especialización tienen una modalidad b-learning= (b-learning).

- La Facultad de Psicología ofrece sus Doctorados, Maestrías y Carreras de Especialización en forma presencial. Las posibilidades de potenciar los aprendizajes con las tecnologías, cuenta con una experiencia desarrollada desde 2006, en el marco de una investigación del Conicet, de una docente-investigadora de dicha Facultad en la carrera de grado. Este 
curso presencial-virtual está destinado a la comisión de sus alumnos y es una experiencia articulada con el Campus Virtual de La UNR, donde la Profesora se desempeña como asesora. Consiste en: "un trabajo de aprendizaje colaborativo: con wikis, foros, con la posibilidad de ofrecerles a los alumnos videos, por ejemplo. Al finalizar el año los estudiantes pidieron que la experiencia se extendiera, con la recomendación a la Facultad de sumar más tecnología" (San Martín, 2008).

- En cuanto al Campus Virtual de la UNR (anteriormente llamado PUNTOEDU), la modalidad educativa mediada por Internet que ofrece incorporó desde el año 2001 la plataforma Moodle, que es una herramienta que posibilita seguir perteneciendo al movimiento de código abierto (open source), compartir con otros docentes e instituciones universitarias nacionales e internacionales, distintas tecnologías con una finalidad educativa, entendiendo que el conocimiento y el aprendizaje se construyen en el marco de una comunidad educativa colaborativa, flexible y cada vez más interactiva.

(...) La decisión de sumar este cambio tecnológico va de la mano de un proceso de investigación desarrollado en el ámbito universitario, y que tiene como primera referencia visible la creación de la Secretaría de Tecnologías Educativas y de Gestión. (...) Entre las ofertas del campus encontramos el área de comunidades. Con sólo acreditar que se es docente de la UNR se le abre el espacio con todas las potencialidades de la plataforma y con el asesoramiento gratuito de especialistas, brindando todas las semanas workshops (talleres) gratuitos para quienes están interesados en conocer la dinámica de interacción y las cuestiones de la didáctica (San Martín, 2008).

También es importante destacar las propuestas de trabajo en talleres educativos que se realizan en el Campus Virtual de la UNR:

Los "Workshops del Campus Virtual UNR" son propuestas de formación integral bajo la modalidad de taller que tienen por finalidad contribuir al diseño conceptual del espacio online de Investigación y Educación en los niveles de pregrado, grado y postgrado. Están destinados a los docentes e investigadores de la UNR, siendo coordinados por los Asesores del Departamento de Educación e Investigación del Campus Virtual (San Martín, 2008).

Durante 2008 y 2009 se llevaron adelante 21 cursos, con un incremento en la matrícula de alumnos de un 150\% en relación al período anterior. En 2009 se graduaron 45 alumnos y del total de inscriptos casi un $70 \%$ finalizó sus estudios. Ello demuestra que la modalidad a distancia no sólo crece, sino que se presenta como una alternativa educativa que promueve índices más altos de retención que la modalidad tradicional. En virtud de ello, la UNR hoy más que 
nunca debe fortalecer las experiencias en EAD y capitalizarlas con una toma de decisiones políticas y académicas que las sistematice a nivel institucional, para que se aplique en las carreras de grado y postgrado de su jurisdicción, con un sistema de evaluación y acreditación que reglamente su funcionamiento.

- En la Facultad de Ciencias Exactas, Ingeniería y Agrimensura, los Doctorados y Maestrías se cursan en forma presencial. Entre las Carreras de Especialización, está Ingeniería Sanitaria a Distancia. Esta facultad fue la pionera en la modalidad de cursado a distancia. Hacia fines de 1990, se propuso incorporar la modalidad de educación a distancia como alternativa a la demanda social de educación en áreas científico-tecnológicas. Como primera medida, se optó por capacitar en la modalidad a distancia al personal de la Facultad a través de un Programa de Capacitación de Postgrado, coordinado académicamente por la Dra. Marta Mena (Especialista en EAD). Durante 1992-1994, se comenzaron a desarrollar actividades a distancia en la Facultad. A partir de este último año, esta Unidad Académica posee un espacio institucional para promocionar y desarrollar actividades en el área de EAD. En la actualidad, el Departamento de Educación a Distancia depende de la Escuela de Postgrado y Educación Continua de la Facultad. Su objetivo principal es la promoción y el desarrollo de actividades que posibiliten la no presencialidad para:

- Actualización y perfeccionamiento docente en los diferentes niveles del Sistema Educativo.

- Educación continua en Ingeniería.

- Formación de postgrado.

- Diseño estratégico que posibilite crear ambientes de aprendizaje más flexibles en el contexto de la enseñanza presencial.

- La Escuela de Postgrado y Educación Continua, además de las Carreras de postgrado de la Facultad, tiene a su cargo la organización de cursos, seminarios, talleres y conferencias de Postgrado, así como las actividades de EAD.

En el Departamento de Educación a Distancia se realizan distintas actividades, entre las que se encuentran: actualización y perfeccionamiento docente para la Educación Secundaria; Capacitación permanente en Ciencias e Ingeniería; Cursos Abiertos a la comunidad; Materiales impresos para el ingreso en carreras universitarias; Implementación de estrategias de EAD en el ámbito de la enseñanza presencial; Capacitación en la modalidad de estudios a distancia (Portal UNR, 2009).

Las Carreras a Distancia que en la actualidad existen presentan las siguientes características: 
Estudio independiente orientado sobre la base del empleo de los materiales escritos en soporte electrónico y de otros recursos relevantes accesibles en sitios seleccionados por los profesores. Obran on-line en formato hipertextual a través del recurso: c-virtual, el Entorno Educativo Virtual de la Facultad. Entorno tutorial permanente para el asesoramiento, el seguimiento y la evaluación de los aprendizajes, combinando intercambios on-line y participación colaborativa en foros. Seguimiento y asistencia permanente del Departamento de Educación a Distancia de la FCEIA en todo lo relativo a los aspectos organizativos y tecnológicos referentes a la modalidad de dictado empleada (Portal UNR, 2009).

Entre las Carreras relevadas pudimos encontrar la Maestría de Recursos Hídricos en zona de llanura y la Especialización de Ingeniería Sanitaria con modalidad en EAD.

La primera y la segunda tienen como duración 2 (dos) años, con un máximo de 4 (cuatro). Su finalidad es la profundización e investigación de los conocimientos sobre la problemática derivada de las transformaciones producidas por la acción del hombre y de la naturaleza en relación con el comportamiento de los recursos hídricos en zona de llanura. La segunda está destinada a la capacitación de los profesionales en problemáticas del saneamiento, vinculada con el diseño, proyecto, operación y control de obras y servicios de abastecimiento de agua y disposición y tratamiento de líquidos cloacales y residuos sólidos urbanos. La Facultad cuenta con Trayectos Curriculares Sistemáticos de Postgrado a distancia (T.C.S.P.):

(...) conjuntos interrelacionados de actividades curriculares de postgrado en torno a un eje temático que corresponde a intereses de la práctica profesional y/o fundamentación teórica, que tienen como finalidad la actualización, capacitación y perfeccionamiento de profesionales". El Trayecto de Postgrado que se cursa con modalidad a distancia es el de "Tratamiento de Aguas y Efluentes", cuya finalidad es "actualizar los conocimientos de los profesionales que trabajan en temas relacionados al tratamiento de aguas (para consumo humano y también residuales) y proporcionar conocimientos científicos a aquellos que se incorporan a esta actividad provenientes de otras disciplinas (Portal UNR, 2009).

La Facultad también cuenta con diferentes cursos a distancia, que contemplan las diversas especialidades que en carreras de grado ofrece. Esta Facultad tiene 90 años de trayectoria en la formación de profesionales y en la vinculación con el medio productivo de la Región.

A través de sus diez carreras de grado forma profesionales altamente capacitados en materia ingenieril, de agrimensura y de las ciencias exactas, 
posibilitando además la formación de postgrado mediante su Escuela de Postgrado y Educación Continua.

Para garantizar la vinculación y la articulación entre el grado, el postgrado, la investigación y la vinculación con el medio, el Consejo Asesor de dicha Escuela está formado por los Directores de Escuela (Carreras de Grado), Directores de Centros y Directores de Institutos. Su organización permite estructurar las carreras de postgrado emergentes de las carreras de grado, así como atender a las demandas del medio en cuanto a carreras de postgrado mono o multidisciplinarias y a trayectos curriculares específicos de capacitación de pre o postgrado. Estas carreras y actividades de postgrado están relacionadas naturalmente con las carreras de grado (Portal UNR, 2009).

En la entrevista realizada a la Dra. Marchisio -responsable del Departamento de Educación a Distancia- sobre el origen de las experiencias en EAD de su Facultad, manifestó:

“(...) empezamos a fines del año '91, con una motivación inicial de la institución por incorporar la EAD; en principio fue en el ámbito del postgrado, la idea fue originaria a partir de la cual se capacitó al personal. Se capacitó gente que era del ámbito del postgrado. Tuvimos una capacitación de dos años. Mientras se hacía esa capacitación, empezaron a gestarse proyectos a partir de un grupo grande de gente, yo de alguna manera coordiné localmente esta actividad, porque la persona que venía a coordinar el Proyecto era de Buenos Aires. Trabajábamos coordinando todos los equipos de todos los docentes que libremente asistieron a enterarse de qué se trataba la EAD. La capacitación duró dos años, trabajé en la gestión de proyectos, el desarrollo de materiales, la compañía, la evaluación de aprendizajes en EAD, en administración educativa a distancia y en ese momento, mientras se fueron gestando los proyectos, fue llevándose la EAD más al ámbito de la articulación con la escuela media. Había muchos grupos de docentes de primer año de las materias de Física y Matemática que se habían incorporado al equipo y que querían (casi todos venían de grupos que estaban trabajando en investigación en educación) acercarse a tomar más conocimiento sobre la modalidad, pero la Secretaría Académica en esa época dio la posibilidad de que se trabajara en la articulación con las Escuela Medias para el ingreso a la Universidad, para aprovechar el potencial de ese grupo docente que estaba interesado en llevar adelante la modalidad, comenzando a desarrollar pequeños proyectos y a elaborar materiales para la EAD, en asignaturas como Álgebra, Análisis Matemático, Física I de las carreras de grado. Con respecto a 
la inclusión de las Nuevas Tecnologías puedo decir que por aquellos tiempos no aplicamos prácticamente nada, todos fueron materiales escritos, recién aparecía el fax como una herramienta de comunicación por medio de la cual, un alumno podía mandar una actividad a través del fax (...). Más tarde el Área de EAD queda afectada a la Escuela de Postgrado y Educación continua de la Facultad. Actualmente dirijo el Área de Postgrado en Educación a Distancia" (Entrevista: 23 de Septiembre de 2009).

Si bien esta entrevista fue realizada en el marco del Proyecto HUM $266 / 2008$, sobre experiencias de EAD en la UNR sobre las carreras de grado, la Profesora reveló algunos datos interesantes para rescatar sobre el inicio de las mismas, con respecto a los postgrados. Las experiencias en esta Facultad, se presentan como innovadoras ya a fines de la década del '90, luego se fueron manteniendo en el tiempo y actualmente desde el Departamento de Postgrado hay varias cátedras que trabajan con espacios virtuales. En general están planificados desde los proyectos de investigación donde trabaja la Dra. Susana Marchisio; otros se están llevando a cabo en la cátedra de Sistemas de Información, con profesores que están trabajando en espacios virtuales, por ejemplo, con Webquest, con blogs, incorporando los conocimientos que se van desarrollando en los seminarios de postgrados.

Solari y Monge (2004) -citando a Litwin (2000)- sostienen en este sentido que:

Las instituciones formadoras de formadores, en este caso específico, las Universidades, deben propiciar ambientes que favorezcan el aprendizaje para que el personal docente aplique los nuevos conocimientos a las actividades docentes que desarrolla. En la sociedad de la información se vislumbra el desarrollo de un sistema educativo y formativo amplio y abierto, que presenta notables potencialidades, pero también fuertes riesgos. En este contexto, si las universidades quieren mantener su rol central en la transmisión de los conocimientos, es necesario que: Identifiquen nuevas políticas de intervención para dar respuestas adecuadas a las necesidades de formación, incrementando la flexibilidad. Reanalicen sus funciones para insertarse en el nuevo contexto y en el nuevo ambiente de la sociedad de la información. Redefinan el rol de sus docentes en función de los nuevos requerimientos para: el profesor universitario, la modalidad a distancia y las nuevas tecnologías. En este marco es evidente que el profesor universitario de los entornos virtuales de enseñanza, también tendrá que redefinir su rol respecto al asumido en el sistema de enseñanza convencional. Las instituciones están cambiando y cambiarán y por tanto el profesor ya no tendrá que estar exclusivamente asociado a una asignatura o ciclo. En este sentido, la figura omnipresente del profesor deja paso al trabajo coordinado del equipo docente que impartirán módulos de cursos online. En la sociedad 
del aprendizaje, nos encontraremos con una enseñanza que recurrirá a las tecnologías interactivas multimedia y a diversos soportes de información (CD-ROM, DVD o CD-R, redes telemáticas).

\section{Algunas reflexiones finales}

El trabajo que presentamos tiene como objetivo fundamental hacer aportes concretos a la UNR sobre la problemática de la Formación Docente en EAD -en carreras de postgrado- y en el uso de las tecnologías de la información y la comunicación con un sentido educativo.

La EAD se presenta en constante crecimiento en este nuevo siglo, favorecida por el desarrollo de medios tecnológicos, y es necesario que los docentes universitarios cuenten con espacios institucionales y académicos, para trabajar y generar propuestas innovadoras.

La modalidad a distancia exige conocimientos que requieren una formación específica de los profesores en la modalidad, disponibilidad de recursos materiales y tecnológicos que deben ser proporcionados por las instituciones que la adoptan. Tiene la particularidad de exigir una organización dinámica y una administración capaz de responder con mayor flexibilidad de tiempo, espacio y autonomía.

La producción investigativa en el campo de la modalidad a distancia plantea permanentemente nuevos desafíos. Desafíos de orden científico, tecnológico y pedagógico-didáctico, que intenten resolver diferentes intereses y vocaciones en el marco de una educación permanente, inclusiva y democratizadora.

Al desarrollo cada vez más masivo de Internet en estos últimos años, se suma en la Educación Superior la necesidad de contar con carreras que ofrezcan sistemas mixtos de cursado o totalmente virtuales.

Las universidades, en general y la UNR en particular, deberían profundizar acciones tendientes al fortalecimiento de carreras de postgrado con experiencias educativas en línea, bajo la denominación de aulas virtuales o campus virtuales. El aula virtual es un entorno de enseñanza y aprendizaje basado en un sistema de comunicación mediado por la computadora, que en la UNR está teniendo un mayor desarrollo en los últimos años.

Por el momento la Facultad que mayor desarrollo tiene en la modalidad es la Facultad de Ciencias Exactas, Ingeniería y Agrimensura (FCEIA). Pero en general no hemos encontrado Facultades que ofrezcan postgrados a distancia. Sería importante que los responsables del Campus Virtual (UNR) propiciaran instancias de trabajo colaborativo con los docentes-investigadores que, a lo largo de las últimas décadas, se han ido formando en esta modalidad y que pueden brindar, no sólo sus saberes teóricos, sino también los prácticos, a través de experiencias muy valiosas, como es el caso de la FCEIA.

El aula virtual permite la interacción de un gran número de estudiantes, quienes estudian, comentan, reflexionan y producen conocimientos en forma 
colaborativa, sin producirse encuentros presenciales. Cabe explicitar que por tecnologías de la información y la comunicación, se entiende al conjunto de procesos y productos derivados de las nuevas herramientas (hardware y software), soportes de la información y canales de comunicación, relacionados con el almacenamiento, procesamiento y transmisión digitalizados de la información.

Parafraseando a Adell y Sales (2000) podemos decir que las computadoras ofrecen una gran cantidad de posibilidades e incluso conectadas en red incrementan su funcionalidad en gran escala. Formando redes, las computadoras no sólo sirven para procesar información almacenada en soportes físicos en cualquier formato digital (discos externos o internos, pendrives, etc.), sino también como herramienta para acceder a información, recursos y servicios prestados por computadoras remotas, como sistema de publicación y difusión de la información y como medio de comunicación entre seres humanos.

El conjunto de aplicaciones informáticas disponibles (correo electrónico, sistemas de discusión en línea, sistemas de videoconferencia, sistemas de audio y video), adecuadamente integrados, serían indispensables y muy flexibles en estrategias didácticas en las carreras de postgrado, para formar los futuros especialistas, maestrandos y doctorandos.

Como afirma Rama (2006), la educación surgió como un servicio presencial y, en el desarrollo de este proceso, nacieron nuevas modalidades de educación y nuevas instituciones. La virtualización y la cultura digital avanzan sin pausar.

Nuestros profesionales, que están cursando Especializaciones, Maestrías y Doctorados, advierten con preocupación la realidad planteada. Creemos que formando capital humano en el campo de la didáctica y en una pedagogía de la virtualidad posibilitaría el desarrollo de las experiencias, para poder dar respuestas concretas a las demandas de una formación docente continua en EAD.

Los comunicadores sociales como los cientistas de la educación -integrantes del equipo- son los más interesados en que se desarrollen líneas de acción al respecto, con políticas académicas que favorezcan el despliegue de la modalidad mediada por las tecnologías.

A nivel local, provincial, nacional, regional e internacional la posibilidad de estudiar Especializaciones, Maestrías y Doctorados en el marco de la EAD, abriría un abanico de posibilidades para todos aquellos sujetos que no pueden asistir regularmente a clases, por múltiples motivos. En la UNR, el desarrollo de la EAD en el campo social y pedagógico dejaría una impronta encomiable, ya que les daría la posibilidad a muchos sectores excluidos, de poder cursar Estudios Superiores de Postgrado pudiendo administrar sus tiempos y espacios de aprendizaje con mayor autonomía y libertad. Como plantea Mena (2005):

(...) modalidad educativa que, mediatizando la mayor parte del tiempo la relación pedagógica entre quienes enseñan y quienes apren- 
den a través de distintos medios y estrategias, permite establecer una particular forma de presencia institucional más allá de su tradicional cobertura geográfica y poblacional, ayudando a superar problemas de tiempo y espacio.

Hay que tener en cuenta las diversas configuraciones que la modalidad fue adoptando en las instituciones de Educación Superior o programas que la desarrollaron. Desde un marco general se pueden armar diversas configuraciones didácticas sobre modelos institucionales en relación a la EAD. La tendencia en las ofertas educativas de la UNR responde a la de las instituciones bimodales, principalmente a través de las ofrecidas por el Campus Virtual, que aún hoy resultan insuficientes. "El modelo bimodal consiste en el desarrollo de un programa o departamento a distancia en el seno de una universidad convencional" (Mena, 2005).

En los '80 la EAD comienza a consolidarse a nivel mundial, lo que permitió su introducción en universidades convencionales dando inicio a la evolución de un modelo bimodal. Esta propuesta trajo cambios y modificaciones, pues se comenzó a dejar de lado el modelo de industrialización y se comenzó a pensar un modelo basado en un paradigma que resignificaba lo contextual, la participación de un alumno activo en la construcción y desarrollo de su aprendizaje. "(...) La multidireccionalidad de la comunicación entre los actores del proceso de enseñanza y aprendizaje" (Mena, 2005).

Este modelo bimodal primó en la EAD hasta finales del siglo XX y aún tiene presencia. No podemos desconocer que en los últimos años comenzamos a presenciar una tendencia hacia la virtualización. Los programas virtuales toman auge con la incorporación de las tecnologías en diversas organizaciones educativas de gestión pública y gestión privada. Las mismas hacen referencia también al desarrollo tecnológico en el diseño de procesos, programas y aplicaciones. Las tecnologías se centran en los procesos de comunicación y podemos agruparlas en tres áreas fundamentales: informática, video y la telecomunicación, con interrelaciones y desarrollos a más de un área.

Desde esta concepción debemos tener presente que la educación virtual es un fenómeno social y complejo. Nos remite a las capacidades de mediación que tienen las tecnologías para producir encuentros formativos, promoviendo así entre los distintos actores (alumnos, docentes, tutores, etc.) nuevos tipos de comunicación y de relaciones socio-culturales.

La Educación virtual es un sistema de formación a distancia que se apoya en las nuevas tecnologías de la comunicación e información (tecnologías, redes de telecomunicación, videoconferencias, TV digital, materiales multimedia, etc.), que combina distintos elementos pedagógicos en un dispositivo que contiene: instancias presenciales, las prácticas, los contactos en tiempo real sincrónicos 
(presenciales, videoconferencias o chats) y los contactos diferidos o asincrónicos (tutores, foros de debate, correo electrónico). Esta nueva concepción formativa utiliza la computadora personal como principal recurso tecnológico, que emplea hipermedia, así como la comunicación digital. Es a partir de estos recursos que la Educación Virtual permite configurar diferentes escenarios formativos que combinados pueden proporcionar un aprendizaje más significativo (Lugo, 2003: 21).

La EAD y virtual en la UNR constituye un área de vacancia que se debe desarrollar más rápidamente, ya que aún no se cuenta con política(s) académica(s) sólidas en este sentido. Esta preocupación debería ocupar el primer lugar en la planificación estratégica, como el gran desafío para este siglo. Preocupa al colectivo docente y a la sociedad en su conjunto, puesto que a nivel mundial se está planteando en todos los foros internacionales la "universalización de la Educación Superior".

Ésta posibilitaría trabajar sobre líneas de acción enmarcadas en una "educación para la sustentabilidad", como favorecedora del acceso de un mayor número de estudiantes al sistema de educación superior, especialmente tratándose de un país latinoamericano como el nuestro. La misma tendría que venir acompañada de un mayor presupuesto para las universidades, a fin de garantizar su democratización. Proponemos la inclusión de la modalidad a distancia y la enseñanza virtual en las carreras de Postgrados, como un aspecto importante a tener en cuenta en el establecimiento de la(s) política(s) académica(s). Esto podría favorecer el trabajo conjunto entre la Escuela de Ciencias de la Educación de la Facultad de Humanidades y Artes, con el Campus Virtual, y las Facultades pioneras (FCEIA) en el desarrollo Seminarios de Formación docente en la modalidad y la oportunidad para establecer un sistema de redes abiertas, que servirían como acceso en la región, de estudiantes de otras Universidades Nacionales y Extranjeras (especialmente las latinoamericanas), mediante un sistema normativo a reglamentar por la UNR.

El objetivo consistiría en operar como un sistema de colaboración entre comunidades educativas virtuales conectadas en la red en la región, articulada a la formación docente continua y flexible. La investigación sobre esta problemática nos permite ir reflexionando sobre el desarrollo de la EAD en las carreras de postgrado de la UNR y sobre la necesidad de establecer y concretar Políticas de Formación Docente, para la formación de capital humano en esta modalidad. Ello requiere de profesionales formados en el campo de sus disciplinas, pero asimismo de las Ciencias de la Educación, especialmente en Currículum, Didáctica/s (general y especiales), Tecnología educativa y Antropología y Sociología de la educación, para poder pensar en sistemas educativos cada vez más democráticos e inclusivos. Reconocemos que a través del 
Campus Virtual se han venido realizando en los últimos 10 años importantes esfuerzos en este sentido.

La modalidad ha crecido en los últimos años en el Nivel Superior y si bien nuestra Universidad ha tenido un proceso lento en aplicarla, lo viene haciendo más en los postgrados que a nivel de carreras de grado. A través del Campus virtual de la UNR se están llevando adelante importantes avances en este campo del conocimiento. Actualmente la UNR cuenta con tres carreras de pre-grado y 21 cursos que se dictan por Internet. El Campus Virtual, desde el 2008, está trabajando conjuntamente con el Programa Dispositivos Hipermediales Dinámicos del Centro Internacional franco Argentino de Ciencias de la Información y de Sistemas (Cifasis) del Conicet en el desarrollo de proyectos de investigación, lo que marca una "maduración en la modalidad". Según la coordinadora del Campus, "la deserción en las propuestas de educación a distancia fue, durante los primeros años del Siglo XXI, una problemática profunda tanto en el campus de la UNR como en otros y a nivel internacional [...] eso tiene que ver con cómo se llevan adelante los procesos de innovación [...] todos estos años permitieron pensar cómo hacer más efectivos los procesos en esta educación mediatizada por la tecnología (San Martín, 2010).

Al finalizar esta investigación la UNR podrá contar con la sistematización de un valioso material documental (que hasta el momento no lo tiene) y con personal docente capacitado y especializado en el Área a Distancia. Esto favorecerá el intercambio con otras Universidades Nacionales y Extranjeras, para ir armando Redes Interinstitucionales en el marco del Consejo Universitario Nacional (CIN).

Para favorecer el desarrollo de este tipo de modalidad la UNR, en el ámbito del CIN, viene realizando algunos aportes significativos con este equipo de investigación, puesto que la Directora del equipo que conformamos, fue invitada por la Secretaría de Ciencia y Tecnología de Rectorado de la UNR, junto a la Directora del Área a Distancia de la FCEIA Dra Susana Marchisio, como representante de la UNR al Taller "TIC en Educación", que se realizó en la ciudad de Santa Fe, en Junio de 2009. Al mismo asistieron además otras Universidades Nacionales como ser: UNL, UBA, UNNE, UNS, UNER, UNP, UNRC, UNSJ; autoridades del Ministerio de Educación de las Provincias de Santa Fe y Entre Ríos, a fin de armar una Red interinstitucional a nivel Nacional.

A nivel Internacional, también se ha constituido con la Facultad de Ciencia Política y RRII una Red de intercambio educativo con la Asociación de Pedagogos Latinoamericanos de Cuba, donde parte del equipo está trabajando sobre esta problemática.

La Escuela de Ciencias de la Educación de la Facultad de Humanidades y Artes, la Escuela de Comunicación Social de la Facultad de Ciencia y Relacio- 
nes Internacionales, la Facultad de Ciencias Exactas, Ingeniería y Agrimensura (Ciclo de Formación Docente), aportan con sus profesionales a través de este proyecto desde una disponibilidad interdisciplinaria en lo epistemológico y metodológico, favoreciendo la realización de un trabajo articulado y colaborativo para la UNR, acorde a los desafíos que demanda la sociedad del conocimiento para desenvolverse en el Siglo XXI.

\section{Referencias Bibliográficas}

- Achilli, E. (1998). La Práctica docente: una interpretación desde los saberes del maestro, en Cuadernos de Formación Docente. Rosario: UNR Editora.

- $\quad$ Barbera, E. y Badia, A. (2004). Educar con aulas virtuales. Madrid: Machado Libros.

- Bolsegui, M. y Fuguet Smith, A. (2006) "Construcción de un modelo conceptual a través de la investigación cualitativa". En Revista Universitaria de Investigación SAPIENS. Vol. $7, N^{\circ} 1$. Caracas (versión digitalizada).

- $\quad$ Carreras De Postgrado (UNR) (1995-2008). Rosario: UNR Editora.

- Congreso Nacional Por Una Educación Superior Democrática Popular, Emancipatoria Y Latinoamericana (2008). Reflexiones del Congreso Nacional por una Educación Superior Democrática, Popular, Emancipadora y Latinoamericana. Córdoba: ADIUC, AGD y COAD.

- Copertari, S. (2007). Tecnología y Educación a distancia desde la complejidad, en Anuario La Trama de la Comunicación (pp. 357-370). Dpto. de Comunicación Social. Facultad de Ciencia Política y RRII. Vol. 11 (Año XI). Rosario: UNR Editora.

- Copertari, S. (2010). La práctica docente universitaria en Educación a Distancia. Procesos metacognitivos y buena enseñanza. Rosario: Laborde editor.

- Doval, L. y Peña, S. (2005). Educación y Nuevas Tecnologías. Módulo 1. Fundamentos y Perspectivas de los recursos pedagógicos y de las TICs en educación, en Materiales de Capacitación directiva en gestión de recursos tecnológicos en la escuela. Universidad Nacional de Catamarca. CAPACYT. Buenos Aires: Formación Docente.

- Fainholc, B. (1999). La interactividad en la Educación a Distancia. Buenos Aires: Paidós.

- Fainholc, B. (2000). Formación del profesorado para el nuevo siglo: Aportes de la tecnología educativa apropiada. Buenos Aires: Lumen.

- $\quad$ Flores, K.E. (2006). Encontrando al profesor virtual, en Revista Mexicana de Investigación Educativa, enero-marzo, Vol. 11, Núm. 28, pp. 91-128.

- Gutiérrez, V., Giovanni, Y. y Orozco, J.C. (2007). Políticas Tecnológicas en un Escenario de Gestión del Conocimiento en Educación Revista Iberoamericana de Educación. № 45, pp. 9-11-19.

- larmusch, S. y Rainoldi, A. (2005). Módulo Tecnologías para la Comunicación Educativa, en Materiales del Seminario de Gestión pedagógica de las TICs. Universidad Nacional de Catamarca. CAPACYT. Buenos Aires: Formación Docente.

- Lago Martinez, S., Gómez Rojas, G. y Mauro, M. (Coord.). (2003). En torno de las metodologías: abordajes cualitativos y cuantitativos. Buenos Aires: Proa XXI.

- $\quad$ Litwin, E., Maggio, M. y Roig, H. (Comp.). (1994). Educación a Distancia en los '90. Desarrollos, problemas y perspectivas. Buenos Aires: Facultad de Filosofía y Letras (UBA) y Programa UBA XXI. 
- Litwin, E. (1997). Las configuraciones didácticas en el marco de una Nueva Agenda Didáctica, en Revista del IICE (pp. 42-51), Buenos Aires.

- Litwin, E. (Comp.) (2000). La educación a distancia. Temas para el debate de una nueva agenda educativa. Buenos Aires: Amorrortu.

- Litwin, E., Maggio, M. y Lipsman, M. (Comps.) (2005). Tecnologías en las aulas. Las nuevas tecnologías en las prácticas de la enseñanza. Casos para el análisis. Buenos Aires: Amorrortu.

- Lion, C. (2006). Imaginar con tecnologías. Relaciones entre tecnologías y conocimiento. Buenos Aires: Stella/La Crujía.

- $\quad$ Lugo, M.T. (Coord.) (2003). Situación presente y perspectivas de desarrollo de los proyectos de Educación Superior Virtual en Argentina. IELSAC. UNESCO.

- Lugo, M.T. (1999). Capacitación a Distancia: acercar la lejanía. Herramientas para el desarrollo de programas a distancia. Buenos Aires: Magisterio Río de la Plata.

- Mapas De Postgrados En Educación Argentina (presencial y a Distancia) Doctorados, Maestrías y Especializaciones. Disponible en: http://mapas.becasyempleos.com. ar/03/03-034.aspx.

- Marquís, C. (2009) Posgrados y Políticas Universitarias. Consideraciones sobre el caso argentino, en Revista RAES, Año 1, Número 1, Noviembre, p. 37.

- Mena, M. (Comp.). (2007). Construyendo la nueva agenda de la Educación a Distancia. Buenos Aires: Crujía.

- Mena, M. (2006). La evolución de la educación a distancia [versión digital]. Disponible en: http://weblog.educ.ar/educacion-tics/cuerpoentrevista.php?idEntrev=50.

- Morin, E. (1995). Introducción al Pensamiento Complejo. Barcelona: Gedisa.

- Pérez, C., (2008). Calidad de la Educación Superior a Distancia en México, documento en línea. Disponible en: http://www.uag.mx/63/a35-03.htm.

- Pérez Lindo, A., Trottini, A. M., Camós, C. y Darín, S. (Coord.). (2005). Gestión del Conocimiento. Un nuevo enfoque aplicable a las organizaciones y la universidad. Buenos Aires: Norma.

- Rama, C. (2006). La Tercera Reforma de la educación superior en América Latina. Buenos Aires: Fondo de Cultura Económica.

- Raposo Villavicencio, M.R. (2004). Fundamentos filosóficos, pedagógicos y psicológicos de la Educación a Distancia en la enseñanza de postgrado. Disponible en: www. monografías.com.

- Sanjurjo, L. (2002). La formación práctica de los docentes. Rosario: Homo Sapiens. Rosario, Diciembre.

- Solari, A. Y Monge, G. (2004). "Un desafio hacia el futuro: Educación a Distancia, Nuevas Tecnologías y Docencia Universitaria”, Universidad Nacional de Río Cuarto. República Argentina, en LatinEduca 2004. com. Primer Congreso Virtual Latinoamericano de Educación a Distancia, 23 de Marzo al 4 de Abril.

- Taylor, S.J. y Bodgam, R. (1992). Introducción a los métodos cualitativos de investigación. Buenos Aires: Paidós.

- Trottini, A.M. (2007). La especificidad de la Gestión Universitaria. Gobierno y Administración de su capital humano. Rosario: UNR Editora. Colección Académica.

- Vega G.R. (2006). La educación continua en México: hacia la transición a la captación a distancia, en Revista Electrónica de Tecnología Educativa, Núm. 20 Enero 2006. Disponible en: http://www.uib.es/depart/gte/gte/edutec e/revelec20/vega20.htm. 


\section{Páginas Web consultadas}

- Facultad de Ciencias Económicas: (http://www.fcecon.unr.edu.ar/posgrado/doctorados/ index.htm. Consultada el 22 de Noviembre de 2009.

- Maestría en Artes (Mención Música y Mención Bellas Artes: (http://www.palermoelectronico. com.ar/modules. php?namefile=article\&sid=34). Consultada el 15 de Febrero de 2010.

- $\quad$ ep_maestrias@fhumyar.unr.edu.ar.Consultada el 3 de Febrero de 2010.

- $\quad$ Campus virtual UNR. www.mesadearena.edu.ar. Consultada el 3 de Febrero de 2010.

- Campus Virtual Puntoedu: www.puntoedu.edu.ar. Consultada el 20 de Febrero de 2010.

- Facultad de Ciencias Exactas, Agrimensura e Ingeniería: http://www.fceia.unr.edu.ar/ inicio/. Consultada el 12 de Noviembre de 2009.

- Diario La Capital on-line. Entrevista a Patricia San Martín Coordinadora del Campus Virtual de la UNR "La UNR ya tiene tres carreras y 21 cursos que se dictan por Internet", 29 de Marzo de 2010. www.lacapital.com.ar.

- Universitas. Periódico Digital de la UNR, Rosario, 17 de Agosto de 2005. Consultado el 9 de Abril de 2010. www.clinica-unr.com.ar. 